\title{
Interaction génotype milieu sur la production laitière chez les bovins II. - Corrélation génétique entre niveaux de production et variation de l'héritabilité
}

\author{
B. BONAITI \\ I.N.R.A., Station de Génétique quantitative et appliquée, Centre de Recherches zootechniques \\ F 78350 Jouy-en-Josas
}

\begin{abstract}
Résumé
Pour étudier l'interaction génotype milieu sur la production laitière, on considère selon la théorie de FALCONER (1952) les expressions du même caractère dans deux milieux comme deux caractères différents mais génétiquement liés. Les données du fichier français de contrôle laitier sont séparées selon l'effet milieu étable en trois groupes auxquels nous associons par hypothèse trois milieux différents. L'héritabilité de la quantité moyenne de matière utile augmente avec le niveau de production $(0,31,0,33$ et 0,42$)$. Les corrélations génétiques entre milieux sont proches ou supérieures à 0,9 . Ces résultats, qui confirment les nombreuses études déjà réalisées sur ce sujet, permettent quelques conclusions pour la sélection laitière en France. Le choix des taureaux sur un index laitier unique est suffisant quel que soit le niveau de production des élevages. Il n'est pas nécessaire d'entretenir des lignées spécialisées pour chaque niveau de production. L'augmentation de précision du testage sur descendance qui pourrait être obtenue en ne réalisant celui-ci que dans les élevages de bon niveau, ne compenserait pas la réduction de capacité de testage qui en résulterait. Aussi faut-il continuer à pratiquer le contrôle sur descendance dans l'ensemble des élevages.
\end{abstract}

\section{Introduction}

En présence d'une interaction génotype $\times$ milieu, la sélection ne porte que sur la composante génétique commune à tous les milieux. Ceci peut, si le phénomène est important, réduire l'efficacité globale du schéma de sélection. L'intérêt réel d'un programme collectif d'amélioration génétique peut même être très limité pour une certaine catégorie d'éleveurs dont le milieu de production s'écarte trop du milieu moyen où est réalisée l'évaluation des reproducteurs. Une première analyse (Bonaiti \& Bertaudière, 1981) révélait, en race bovine Frisonne, que l'augmentation réelle de production de lait liée à l'utilisation d'un taureau reconnu améliorateur est d'autant plus sensible que les conditions de milieu sont plus favorables. Cette deuxième étude tente de cerner plus finement ce phénomène à partir de l'estimation de l'héritabilité de la production laitière selon le milieu de production et des corrélations génétiques entre ces milieux selon la méthode proposée par FaLCONER (1952). Une corrélation 
génétique plus petite que 1 montrerait que le classement des taureaux peut varier avec le niveau de production des étables. Une variation de l'héritabilité laisserait supposer que l'expression du génotype d'une génisse est plus importante dans un milieu que dans un autre.

\section{Matériel et méthodes}

En France, la gestion des données du contrôle laitier et l'organisation des calculs des indices de sélection (Poutous et al., 1981) nous permettent de disposer très facilement d'un fichier des données de production laitière corrigées pour l'ensemble des effets autres que les effets taureau (étable $\times$ année, saison de vêlage, numéro de lactation, valeur génétique de la mère...). Cette analyse ne porte que sur les premières lactations de génisses de race Française Frisonne ayant vêlé entre janvier 1977 et septembre 1979. La variable analysée est la quantité moyenne des matières utiles (MMU), principale variable servant à l'indexation des reproducteurs. Elle est définie en fonction de la quantité de lait (QL) des taux butyreux (TB) et protéique (TP) selon la formule :

$$
\mathrm{MMU}=\mathrm{QL} \times \frac{\mathrm{TB}+1,21 \mathrm{TP}}{2}
$$

Pour assurer une plus grande variabilité génétique entre pères et surtout des effectifs et une répartition des filles dans les étables indépendants de la valeur du taureau, le fichier est limité aux seules performances des taureaux de testages nés entre 1970 et 1974. Ce fichier de données est donc identique à celui déjà présenté par Bonaiti \& BerTaudière (1981). Toutefois, les lactations qui, lorsque ce fichier fut prélevé dans la chaîne d'indexation nationale, n'étaient pas encore terminées et dont la production totale était obtenue par extrapolation, sont éliminées de cette deuxième analyse.

Pour étudier l'interaction génotype $\times$ milieu, nous considérons selon la théorie de Falconer (1952), les expressions du même caractère dans deux milieux comme deux caractères différents mais génétiquement liés. Pour deux milieux différents, nous avons deux phénotypes $P_{1}$ et $P_{2}$, deux valeurs génétiques additives $G_{1}$ et $G_{2}$ et deux effets milieu $M_{1}$ et $M_{2}$ :

$$
\begin{aligned}
& P_{1}=G_{1}+M_{1} \\
& P_{2}=G_{2}+M_{2}
\end{aligned}
$$

Pour chacun de ces deux caractères, il est possible d'estimer l'héritabilité :

$$
h_{1}^{2}=\frac{\operatorname{Var}\left(G_{1}\right)}{\operatorname{Var}\left(P_{1}\right)} \quad h_{2}^{2}=\frac{\operatorname{Var}\left(G_{2}\right)}{\operatorname{Var}\left(P_{2}\right)}
$$

Bien que les deux caractères $P_{1}$ et $P_{2}$ ne soient pas mesurables sur le même individu, il est cependant possible d'estimer la corrélation génétique $R\left(G_{1}, G_{2}\right)$.

Les estimations des effets étables (Poutous et al., 1981), qui sont obtenues lors du calcul des indices de sélection, permettent de distinguer parmi les élevages soumis au contrôle laitier trois groupes correspondant à trois milieux différents : 
Milieu

Bas (B)

Moyen (M)

Haut $(\mathrm{H})$
Effet étable ( $k g$ de matière utile)

$$
\begin{aligned}
& \text { de }-50 \text { à }-10 \\
& \text { de }-10 \text { à }+10 \\
& \text { de }+10 \text { à }+50
\end{aligned}
$$

Cette classification diffère de celle qui pourrait être faite en fonction des moyennes de production : l'effet étable, qui est indépendant du génotype moyen des animaux du troupeau, représente la valeur de l'effet sur la production laitière du milieu propre à une étable alors que la moyenne des productions correspond à l'action conjuguée du génotype et du milieu.

Les estimations des variances et covariances génétiques reposent sur le modèle interprétatif suivant :

$$
\mathrm{Y}_{\mathrm{mijk}}=\mu_{\mathrm{m}}+\mathrm{GP}_{\mathrm{mi}}+\mathrm{P}_{\mathrm{mij}}+\mathrm{E}_{\mathrm{mijk}}
$$

où $\mathrm{Y}_{\mathrm{mijk}}$ est la performance réalisée dans le milieu $\mathrm{m}$ par la kième fille du jième père issu du iième grand-père.

$\mu_{\mathrm{m}} \quad$ est l'effet moyen propre au milieu $\mathrm{m}$.

$\mathrm{GP}_{\mathrm{mi}}$ est une variable aléatoire représentant l'effet propre du jième grand-père dans le milieu $\mathrm{m}$.

$P_{\text {mij }}$ est une variable aléatoire représentant l'effet propre du jième père issu $\mathrm{du} \mathrm{i}^{\mathrm{ième}}$ grand-père dans le milieu $\mathrm{m}$.

$E_{m i j k}$ est une variable aléatoire représentant l'effet résiduel de la kième fille du jième père issu du iième grand-père dans le milieu $\mathrm{m}$.

La loi du vecteur des variables alêatoires $G P_{m i}, P_{m i j}, E_{m i j k}$, est multi-normale centrée de matrice de covariance :

\begin{tabular}{ccccccc}
\hline \hline & $\mathrm{GP}_{\mathrm{m}}$ & $\mathrm{P}_{\mathrm{m}}$ & $\mathrm{E}_{\mathrm{m}}$ & $\mathrm{GP}_{\mathrm{m}}$, & $\mathrm{P}_{\mathrm{m}}$, & $\mathrm{E}_{\mathrm{m}} \cdot$ \\
\hline $\mathrm{GP}_{\mathrm{m}}$ & $\sigma_{\mathrm{GP}_{\mathrm{m}}}^{2} \cdot \mathrm{I}$ & $\underline{\mathrm{O}}$ & $\underline{\mathrm{O}}$ & $\operatorname{cov}\left(\mathrm{GP}_{\mathrm{m}}, \mathrm{GP}_{\mathrm{m}}\right) \cdot \mathrm{I}$ & $\underline{\mathrm{O}}$ & $\underline{\mathrm{O}}$ \\
$\mathrm{P}_{\mathrm{m}}$ & $\underline{\mathrm{O}}$ & $\sigma_{\mathrm{P}_{\mathrm{m}}^{2}}^{2} \mathrm{I}$ & $\underline{\mathrm{O}}$ & $\underline{\mathrm{O}}$ & $\operatorname{cov}\left(\mathrm{P}_{\mathrm{m}}, \mathrm{P}_{\mathrm{m}}\right) \cdot \mathrm{I}$ & $\underline{\mathrm{O}}$ \\
$\mathrm{E}_{\mathrm{m}}$ & $\underline{\mathrm{O}}$ & $\underline{\mathrm{O}}$ & $\sigma_{\mathrm{E}_{\mathrm{m}}}^{2} \mathrm{I}$ & $\underline{\mathrm{O}}$ & $\underline{\mathrm{O}}$ & $\underline{\mathrm{O}}$ \\
\hline \hline
\end{tabular}

où I représente la matrice unité et $\underline{\mathrm{O}}$ la matrice nulle.

Les estimations des paramètres $\hat{\sigma}_{\mathrm{GP}_{\mathrm{m}}}^{2}$, $\hat{\sigma}_{\mathrm{P}_{\mathrm{m}}}^{2}$, et $\hat{\sigma}_{\mathrm{E}_{\mathrm{m}}}^{2}$ sont obtenues par la méthode I d'Henderson (1953) de façon indépendante pour chaque milieu. Les estimations des covariances entre milieu sont obtenues selon une méthode décrite par MALLARD et al. (1983) à partir de deux sommes de coproduits :

$$
\begin{aligned}
& s_{\mathrm{a}}=\Sigma_{\mathrm{i}} k_{\mathrm{i}}\left(\mathrm{Y}_{\mathrm{mi} . .}-\mathrm{Y}_{\mathrm{m} . .}\right)\left(\mathrm{Y}_{\mathrm{m}^{\prime} \mathrm{i} . .}-\mathrm{Y}_{\mathrm{m}^{\prime} \ldots}\right) \\
& \mathrm{s}_{\mathrm{b}}=\boldsymbol{\Sigma}_{\mathrm{ij}} \mathrm{k}_{\mathrm{ij}}\left(\mathrm{Y}_{\mathrm{mij} .}-\mathrm{Y}_{\mathrm{mi} . .}\right)\left(\mathrm{Y}_{\mathrm{m}^{\prime} \mathrm{ij} .}-\mathrm{Y}_{\mathrm{m}^{\prime} \mathrm{i} . .}\right)
\end{aligned}
$$

avec $k_{i}=\sqrt{N_{m i} N_{m^{\prime} i}}$ et $k_{i j}=\sqrt{N_{m i j} N_{m}{ }^{\prime i j}}$ 
où $\left(\mathrm{Y}_{\mathrm{m} \ldots}, \mathrm{Y}_{\mathrm{mi} . .}, \mathrm{Y}_{\mathrm{mij}}, \mathrm{N}_{\mathrm{m}}, \mathrm{N}_{\mathrm{mi}}, \mathrm{N}_{\mathrm{mij}}\right.$ représentent respectivement les moyennes des performances et les effectifs correspondants aux indices $m, m_{i}, m_{i j}$ ).

En définissant :

$$
\begin{aligned}
& \lambda_{1}=\Sigma_{\mathrm{ij}} \mathrm{k}_{\mathrm{ij}}\left(1-\frac{\mathrm{N}_{\mathrm{mij}}}{\mathrm{N}_{\mathrm{mi}}}-\frac{\mathrm{N}_{\mathrm{m}^{\prime} \mathrm{ij}}}{\mathrm{N}_{\mathrm{m}^{\prime} \mathrm{i}}}+\Sigma_{\mathrm{j}} \frac{\mathrm{N}_{\mathrm{mij}} \mathrm{N}_{\mathrm{m}^{\prime} \mathrm{ij}}}{\mathrm{N}_{\mathrm{mi}} \mathrm{N}_{\mathrm{m}^{\prime} \mathrm{i}}}\right) \\
& \lambda_{2}=\Sigma_{\mathrm{i}} \mathrm{k}_{\mathrm{i}}\left(\frac{\Sigma_{\mathrm{j}} \mathrm{N}_{\mathrm{mij}} \mathrm{N}_{\mathrm{m}^{\prime} \mathrm{ij}}}{\mathrm{N}_{\mathrm{mi}} \mathrm{N}_{\mathrm{m}^{\prime} \mathrm{i}}}-\frac{\Sigma_{\mathrm{j}} \mathrm{N}_{\mathrm{mij}} \mathrm{N}_{\mathrm{m}^{\prime} \mathrm{ij}}}{\mathrm{N}_{\mathrm{mi}} \mathrm{N}_{\mathrm{m}^{\prime}}}-\frac{\Sigma_{\mathrm{j}} \mathrm{N}_{\mathrm{mij}} \mathrm{N}_{\mathrm{m}^{\prime} \mathrm{ij}}}{\mathrm{N}_{\mathrm{m}} \mathrm{N}_{\mathrm{m}^{\prime} \mathrm{i}}}+\frac{\Sigma_{\mathrm{ij}} \mathrm{N}_{\mathrm{mij}} \mathrm{N}_{\mathrm{m}^{\prime} \mathrm{ij}}}{\mathrm{N}_{\mathrm{m}} \mathrm{N}_{\mathrm{m}}{ }^{\prime}}\right) \\
& \lambda_{3}=\Sigma_{\mathrm{i}} \mathrm{k}_{\mathrm{i}} \quad\left(1-\frac{\mathrm{N}_{\mathrm{mi}}}{\mathrm{N}_{\mathrm{m}}}-\frac{\mathrm{N}_{\mathrm{m}^{\prime} \mathrm{i}}}{\mathrm{N}_{\mathrm{m}} \cdot}+\frac{\Sigma_{\mathrm{i}} \mathrm{N}_{\mathrm{mi}} \mathrm{N}_{\mathrm{m}}{ }^{\prime} \mathrm{i}}{\mathrm{N}_{\mathrm{m}} \mathrm{N}_{\mathrm{m}}}\right)
\end{aligned}
$$

les estimations sont données par :

$$
\begin{gathered}
\widehat{\operatorname{cov}\left(G P_{m}, G P_{m^{\prime}}\right)}=\frac{s_{a}}{\lambda_{1}} \\
\widehat{\operatorname{cov}\left(P_{m i j}, P_{m^{\prime} \mathrm{ij}}\right)}=\frac{s_{b}-\frac{\lambda_{2}}{\lambda_{1}} s_{a}}{\lambda_{3}}
\end{gathered}
$$

Les variances génétiques additives $\left(\hat{\sigma}_{\mathrm{A}_{\mathrm{m}}}^{2}\right)$ et phénotypiques $\left(\hat{\sigma}_{\mathrm{Y}_{\mathrm{m}}}^{2}\right)$ propres à chaque milieu et la covariance génétique $\widehat{\operatorname{cov}\left(\mathrm{A}_{\mathrm{m}}, \mathrm{A}_{\mathrm{m}}\right)}$ entre milieux sont obtenues à partir des estimations précédentes :

$$
\begin{aligned}
& \hat{\sigma}_{A_{m}}^{2}=4\left(\hat{\sigma}_{G P_{m}}^{2}+\hat{\sigma}_{P_{m}}^{2}\right) . \\
& \hat{\sigma}_{Y_{m}}^{2}=\left(\hat{\sigma}_{G P_{m}}^{2}+\hat{\sigma}_{P_{m}}^{2}+\hat{\sigma}_{E_{m}}^{2}\right) \\
& \widehat{\operatorname{cov}\left(A_{m}, A_{m}\right)}=4 \widehat{\left[\operatorname{cov}\left(G P_{m}, G P_{m^{\prime}}\right)\right.}+\widehat{\left.\operatorname{cov}\left(P_{m}, P_{m^{\prime}}\right)\right]}
\end{aligned}
$$

Les héritabilités et les corrélations génétiques sont ensuite estimées par :

$$
\hat{h}_{m}^{2}=\frac{\hat{\sigma}_{A_{m}}^{2}}{\hat{\sigma}_{Y_{m}}^{2}} \quad \text { et } \quad \hat{R}_{m m^{\prime}}=\frac{\operatorname{cov} A_{m}, A_{m^{\prime}}}{\left(\hat{\sigma}_{A_{m}}^{2} \hat{\sigma}_{A_{m}}^{2}\right)^{1 / 2}}
$$

Les écarts types d'erreur d'échantillonnage sont calculés en supposant les effectifs équilibrés pour les estimations de $\hat{\sigma}_{\mathrm{GP}_{\mathrm{m}}}^{2}, \hat{\sigma}_{\mathrm{P}_{\mathrm{m}}}^{2}$ et $\hat{\sigma}_{\mathrm{E}_{\mathrm{m}}}^{2}$ et selon la méthode décrite par Mallard et al. (1983) pour les covariances entre milieux.

Nos calculs ne prennent en compte que les données relatives à des taureaux présentant dix descendants au moins dans chacun des trois milieux. L'échantillon de descendances ainsi défini est, pour chaque groupe d'étables, représentatif de l'ensemble des données vis-à-vis de la valeur génétique estimée des taureaux. Grâce à cet échantillonnage il est peu vraisemblable que les comparaisons entre les valeurs des variances génétiques ou de l'héritabilité selon le groupe d'étables, soient faussées par une inégale répartition des filles. 


\section{Résultats}

Les estimations des composantes de la variance sont présentées dans le tableau 1.

Ils font apparaître une augmentation des variances avec le niveau de production. En valeur relative, la variance génétique $\left(\sigma_{\mathrm{A}_{m}}^{2}\right)$ augmente plus que celle des effets résiduels. Et c'est la composante grand-père qui constitue la principale cause de l'augmentation de la variance génétique. L'écart de valeur des variances génétiques entre les milieux $\mathrm{M}$ et $\mathrm{H}$ est le triple de celui qui est observé entre les milieux B et $\mathbf{M}$. L'évolution de l'héritabilité suit celle de la variance génétique puisque celle-ci augmente plus que celle des effets résiduels. Mais si l'augmentation de ce critère est très marquée entre les milieux $M$ et $H(0,33$ à 0,42$)$, elle est par contre très minime entre les milieux $\mathrm{B}$ et $\mathrm{M}(0,31$ à 0,33$)$.

\section{TABLEAU 1}

Estimation des composantes de la variance et des paramètres génétiques $\left(\sigma_{A m}^{2}\right.$ et $\left.h^{2}{ }_{m}\right)$ selon le groupe d'étables.

Estimation of variance components and genetic parameters $\left(\sigma^{2}\right.$ Am and $\left.h^{2} m\right)$ according to farm groups.

\begin{tabular}{|c|c|c|c|c|c|c|c|}
\hline & & \multicolumn{6}{|c|}{ Groupe d'étables } \\
\hline & & \multicolumn{2}{|r|}{ Bas } & \multicolumn{2}{|r|}{ Moyen } & \multicolumn{2}{|r|}{ Haut } \\
\hline & & d.1. & variance & d.l. & variance & d.l. & variance \\
\hline $\begin{array}{c}\text { Source } \\
\text { de } \\
\text { variation }\end{array}$ & $\begin{array}{l}\text { Grand-père } \ldots \\
\text { Père } \ldots \ldots \ldots \\
\text { Résiduelle } \ldots\end{array}$ & $\begin{array}{r}266 \\
723 \\
21230\end{array}$ & $\begin{array}{r}32,5 \pm 5,7 \\
56,3 \pm 5,6 \\
1060,6 \pm 10,3\end{array}$ & $\begin{array}{r}266 \\
723 \\
26265\end{array}$ & $\begin{array}{r}45,4 \pm 6,8 \\
59,0 \pm 5,5 \\
1152,1 \pm 10,0\end{array}$ & $\begin{array}{r}266 \\
723 \\
27994\end{array}$ & $\begin{array}{c}82,4 \pm 10,3 \\
66,3 \pm 6,0 \\
1261,1 \pm 10,7\end{array}$ \\
\hline \multicolumn{2}{|c|}{$\begin{array}{l}\text { Variance génétique }\left(\sigma^{2}{ }_{\mathrm{Am}}\right) \\
\text { Héritabilité }\left(\mathrm{h}^{2}{ }_{\mathrm{m}}\right) \ldots \ldots\end{array}$} & \multicolumn{2}{|c|}{$\begin{array}{c}355,2 \\
0,31 \pm 0,02\end{array}$} & \multicolumn{2}{|c|}{$\begin{array}{c}417,5 \\
0,33 \pm 0,02\end{array}$} & \multicolumn{2}{|c|}{$\begin{array}{c}594,5 \\
0,42 \pm 0,03\end{array}$} \\
\hline
\end{tabular}

Les écarts types des estimées des diverṣ paramètres sont donnés dans le tableau 1. Pour comparer deux estimations du même paramètre obtenues dans deux milieux différents, il faudrait tenir compte aussi du fait que celles-ci sont obtenues avec le même échantillon de pères. Ceci augmente probablement beaucoup la précision de la comparaison de deux estimations différentes pour l'héritabilité ou pour la variance des effets père. Pour cette raison, les estimations correspondantes aux milieux $\mathbf{M}$ et $\mathrm{H}$ sont vraisemblablement différentes entre elles.

Les covariances et les corrélations sont données dans le tableau 2.

Les corrélations génétiques $\left(\mathrm{R}_{\mathrm{mm}}\right)$ sont supérieures à 0,86 . En calculant les corrélations génétiques avec la seule composante père plutôt qu'avec la somme des composantes père et grand-père, on observe des valeurs très proches de celles qui sont données dans le tableau 2. Les corrélations entre le milieu intermédiaire et les milieux $\mathrm{B}$ ou $\mathrm{H}$ sont plus importantes. La corrélation $\mathrm{B}-\mathrm{H}$ est légèrement inférieure au produit des corrélations B-M et $\mathrm{M}-\mathrm{H}$. 
TABLEAU 2

Estimation des composantes de la covariance et des covariances (cov $A_{m} A_{m}$ ) et corrélations $\left(R_{m m^{\prime}}\right)$ génétiques entre groupes d'étables.

Estimation of covariance components and genetic covariance ( $\left.\operatorname{cov} A_{m} A_{m^{\prime}}\right)$ and correlation $\left(R_{m m^{\prime}}\right)$ between group of farms.

\begin{tabular}{|c|c|c|c|c|}
\hline \multicolumn{2}{|r|}{ Milieux } & \multirow{2}{*}{$\begin{array}{c}\mathrm{B} \times \mathrm{M} \\
36,8 \pm 8,6 \\
52,6 \pm 5,0\end{array}$} & \multirow{2}{*}{$\begin{array}{c}\mathrm{B} \times \mathrm{H} \\
47,1 \pm 11,0 \\
51,8 \pm 5,2\end{array}$} & \multirow{2}{*}{$\begin{array}{c}\mathrm{M} \times \mathrm{H} \\
61,3 \pm 13,7 \\
61,2 \pm 5,6\end{array}$} \\
\hline $\begin{array}{c}\text { Source } \\
\text { de variation }\end{array}$ & $\begin{array}{l}\text { Grand-père } \ldots \ldots \\
\text { Père } \ldots \ldots \ldots\end{array}$ & & & \\
\hline \multicolumn{2}{|c|}{$\begin{array}{l}\text { Covar. génét. } \operatorname{Cov} A_{m} A_{m^{\prime}} \ldots \ldots \ldots \\
\text { Correl. génét. } R_{m m^{\prime}} \ldots \ldots \ldots \ldots\end{array}$} & $\begin{array}{c}357,3 \pm 37,5 \\
0,93\end{array}$ & $\begin{array}{c}395,3 \pm 46,7 \\
0,86\end{array}$ & $\begin{array}{c}490,0 \pm 57,6 \\
0,98\end{array}$ \\
\hline
\end{tabular}

\section{Discussion}

\section{A. Paramètres génétiques selon le milieu de production}

Nos résultats peuvent être comparés aux résultats bibliographiques déjà publiés sur le même sujet: Gravert (1959), Mason \& Robertson (1956), Robertson et al. (1960), Mitchell et al. (1961), Legates (1962), Van Vleck (1963), Van Vleck \& Bradford (1964), Syrstad (1964), Burnside \& Rennie (1968), Norman et al. (1972), Averdunk \& AlPS (1971) et DANELL (1982). D’une façon générale on observe une légère diminution du coefficient de variation avec le niveau de production tandis que les variances génétiques et résiduelles augmentent. En valeur relative, la variance des effets résiduels augmente autant que le niveau moyen de production. L'augmentation de la variance des effets père est en valeur relative au moins le double de celle du niveau de production. Ceci doit donc entraîner logiquement une augmentation de l'héritabilité avec le niveau de production. C'est effectivement le cas pour MASON \& RoBertson (1956), Van Vleck (1963), Syrstad (1964), Norman et al. (1972), Averdunk \& Alps (1971) et Danell (1982). Pour Robertson et al. (1960), l'héritabilité présente un optimum pour le milieu intermédiaire. Et pour BuRNSide \& RENNIE (1968), si l'héritabilité croît sur les six premières classes de milieu, en revanche elle présente une plus faible valeur pour la dernière classe. Pour Legates (1962), qui prend pour estimation le double de la corrélation mère-fille, l'héritabilité est maximum pour les classes intermédiaires. On pourrait d'ailleurs rapprocher ce dernier résultat de ceux de Mrtchell et al. (1961) et de VAN Vleck \& BRADFord (1964) qui calculent l'héritabilité par la même méthode et observent aussi une valeur maximum pour la classe intermédiaire. GRAVERT (1959), observe, sur le même fichier, une élévation de l'héritabilité avec la méthode mère-fille et une stabilité avec la méthode des demi-sueurs. Mais il ne semble pas, selon V VAN VLECK (1964), que ce type d'évolution de l'héritabilité puisse être relié avec la methode d'estimation utilisée. Ce dernier auteur obtient lui-même un résultat inverse.

Nos résultats obtenus sur le fichier français du contrôle laitier sont donc conformes à la plupart des résultats déjà publiés sur le même sujet. Cependant, selon la bibliographie l'augmentation de l'héritabilité est, d'une manière générale, plus nette entre le milieu bas et le milieu intermédiaire. Au contraire, nos résultats montrent une plus 
forte élévation entre les milieux intermédiaire et haut. Nous n'avons pas d'élément pour expliquer cette divergence.

On doit donc s'attendre à une élévation de l'héritabilité, peut-être d'au moins 0,10 en valeur absolue, lorsque le niveau de production augmente. Cette tendance, qui est liée à l'augmentation de la variance des effets père, suggère qu'une augmentation du niveau de production permet une meilleure extériorisation des différences génétiques entre taureaux. Il n'est évidemment pas possible avec nos résultats d'en donner une explication précise. Ceci peut provenir d'un niveau d'alimentation insuffisant dans certains élevages pour exploiter complètement les potentialités génétiques des génisses issues des meilleurs taureaux. Un rationnement individuel selon la production peut conduire aussi à une augmentation des écarts de production entre animaux de potentiels génétiques différents. On peut penser aussi que l'amélioration du contrôle de l'environnement dans les meilleurs élevages entraîne une réduction de la variance des effets milieux et que les effets résiduels, qui en dépendent largement, présentent malgré des phénomènes d'échelle, une moindre augmentation de leur variance que les effets pères.

\section{B. Corrélations génétiques entre milieux de production}

Les valeurs des corrélations génétiques entre groupes d'élevages indiquent que la variabilité de la production laitière correspond pour une grande part à l'expression d'un même caractère génétique quel que soit le niveau de production et confirment ainsi, pour la situation particulière de l'élevage français, les conclusions qui pouvaient être tirées des études étrangères déjà réalisées sur ce même problème : MASON \& Robertson (1956), Robertson et al. (1960), Van Vleck (1963), Syrstad (1964), Burnside \& Rennie (1968), Mc Daniel \& Corley (1967), Averdunk \& Alps (1971), Danell (1982). Ainsi les valeurs des corrélations génétiques observées entre niveaux de production étaient supérieures à 0,85 et même 0,90 pour la plupart.

On peut ajouter que d'autres facteurs de classification que le niveau de production du troupeau furent considérés par d'autres auteurs: la saison de vêlage (BRANTON et al., 1974), le régime alimentaire (MAO \& Burnside, 1969; Richardson, 1970 ; Wiggans \& Van Vleck, 1978), le type d'habitat (Burdick \& Mc Guillard, 1963 ; MaO \& Burnside, 1969 ; WigGans \& VAN Vleck, 1970), la situation géographique (Lytton \& Legates, 1966 ; Norman et al., 1972 ; Petersen, 1975 ; Mc Dowell et al., 1976). Dans l'ensemble ces travaux ne révèlent pas d'interaction notable entre l'effet taureau et le facteur milieu considéré. MAO \& BURNSIDE (1969) observent que, parmi les neuf facteurs de classification considérés par eux, seule l'importance du concentré dans la ration d'été présente un effet d'interaction notable avec les effets génétiques. LytTon \& Legates (1966), Wiggans \& VAN VLeck (1979 et 1978) observent de fortes valeurs de la corrélation génétique respectivement entre régions, types d'habitat et régimes alimentaires. Mc DOwell et al. (1976) observent pour des taureaux utilisés d'une part aux U.S.A. et au Canada et d'autre part au Mexique, mais peut-être dans des conditions relativement favorables, de bonnes corrélations $(0,61$ et 0,81$)$ entre les valeurs génétiques estimées de part et d'autre. Petersen (1975), en analysant des résultats de descendance obtenus au Danemark et en Europe Centrale, observe des valeurs d'héritabilité comparables et une forte corrélation génétique entre les deux situations géographiques. 


\section{Conséquences pour le contrôle de descendance}

Les covariances génétiques entre milieux de production permettent de préciser ce qui peut être réellement espéré du contrôle de descendance des taureaux. Supposons qu'il existe trois milieux de production et trois caractères différents comme nous l'avons fait pour notre analyse. Les données du testage proviennent de manière équivalente de ces trois milieux de production. L'indice de sélection des taureaux correspond donc à l'estimation d'une valeur génétique moyenne $\mathbf{G}$, défini par la combinaison de trois valeurs génétiques différentes :

$$
\mathrm{G}=\frac{1}{3}\left(\mathrm{G}_{\mathrm{B}}+\mathrm{G}_{\mathrm{M}}+\mathrm{G}_{\mathrm{H}}\right)
$$

Les covariances et corrélations entre $G$ d'une part et $G_{B}, G_{M}$ et $G_{H}$ d'autre part peuvent être calculées en utilisant les estimations présentées dans les tableaux 1 et 2 pour les variances et covariances génétiques (tabl. 3).

\section{TABleau 3}

Covariance et corrélation entre la valeur génétique moyenne $(G)$ et les valeurs génétiques $G_{B}, G_{M}, G_{H}$ propres à chacun des trois milieux.

Covariance and correlation between mean genetic value $(G)$ and the genetic value $G_{B}, G_{M}, G_{H}$ of the three environments.

\begin{tabular}{c|c|c|c}
\hline Milieu $\mathrm{m}:$ & Bas & Moyen & Haut \\
\hline $\operatorname{Cov}\left(\mathrm{G}, \mathrm{G}_{\mathrm{m}}\right) \ldots \ldots \ldots \ldots$ & 369,3 & 421,6 & 493,3 \\
\hline $\mathrm{R}_{\mathrm{G}, \mathrm{Gm}} \ldots \ldots \ldots \ldots$ & 0,95 & 1,00 & 0,98 \\
\hline
\end{tabular}

L'indice de sélection permet donc de mesurer une valeur génétique moyenne (G) très proche de celle $\left(G_{B}, G_{M}\right.$ ou $\left.G_{H}\right)$ qui est en rapport avec la production de lait dans un milieu particulier puisque les corrélations génétiques sont toutes très élevées. Mais l'espérance, connaissant l'estimation (indice de sélection) de la valeur génétique globale (G) d'un taureau, de la future performance d'une de ses filles dans un milieu donné dépend du coefficient de régression des performances sur $G$ et varie avec le milieu ( $\mathrm{B}, \mathrm{M}$ ou $\mathrm{H}$ ) comme la covariance entre les valeurs génétiques $\mathrm{G}$ d'une part et $G_{B}, G_{M}$ ou $G_{H}$ d'autre part.

La supériorité espérée de production des filles des taureaux d'indices de sélection plus élevés est donc sensiblement plus grande dans les élevages de milieu plus favorable. Ceci correspond aux conclusions tirées de la comparaison entre classes d'étables des valeurs du coefficient de régression des performances sur l'indice du père (Bonaiti \& Bertaudière, 1982).

\section{Conclusion}

Ces résultats ainsi que ceux déjà présentés par Bonaiti \& Bertaudière (1981) apportent des réponses relativement claires à plusieurs questions posées par les producteurs de lait et les sélectionneurs. Pour les premiers, l'important est de savoir 
si le choix des mâles sur l'index laitier suffit pour l'amélioration génétique de leur cheptel, sans qu'il soit nécessaire de tenir compte du niveau moyen de production de leur étable. Notre réponse sur ce point est affirmative. Cependant nous avons observé que, dans les limites, il est vrai, relativement étroites de notre fichier, l'augmentation réelle de production liée à l'utilisation d'un taureau reconnu améliorateur sera d'autant plus sensible que les conditions de milieu seront plus favorables (Bonaitı \& Bertaudière, 1981). Cette variabilité de réponse est liée, selon ces derniers résultats, à des possibilités d'extériorisation d'un même caractère génétique plus importantes dans un bon milieu plutôt qu'à la présence de caractères génétiques partiellement différents et propres à un milieu particulier.

Pour les sélectionneurs, il n'est pas indispensable d'entretenir des lignées spécialisées pour tel ou tel niveau d'élevage puisque c'est en grande partie le même caractère génétique qui s'exprime quel que soit le milieu de production. Par ailleurs, en reprenant la théorie de HAMmOND (1947) qui semble dans le contexte français s'appliquer à la production laitière, on pourrait ne réaliser le contrôle de descendance que dans les meilleurs élevages puisque, tout en obtenant un classement des taureaux valable quel que soit le niveau de production, on réaliserait un testage plus précis. Mais les différences d'héritabilité selon le groupe d'élevage ne sont pas suffisamment importantes pour entraîner un gain de précision qui puisse compenser les conséquences d'une réduction de la capacité de testage. Tout au plus, pourrait-on proposer d'éliminer les données issues des élevages vraiment très mauvais.

Une autre argumentation nous aurait de toute manière empêché de reprendre la proposition de Hammond (1947). Notre étude ne porte que sur l'un, le principal peutêtre, des éléments concernés par l'économie de la production laitière. Une interaction plus sensible pourrait exister entre le génotype et le milieu pour d'autres caractères : reproduction, longévité. Pour pouvoir la mesurer et éventuellement en tenir compte, il semble préférable de poursuivre la politique actuelle de testage sur descendance dans la gamme de milieux la plus représentative possible de l'élevage français ; objectif peut-être impossible à atteindre en totalité dans la mesure où le niveau des élevages soumis au contrôle laitier est supérieur à celui des autres.

Reçu pour publication en mars 1982.

\section{Remerciements}

Nous remercions B. BiBE \& G. MALLARD pour leurs remarques intéressantes et utiles sur ce manuscrit.

\section{Summary}

Genotype by environment interaction on cattle dairy production II. - Genetic correlation between production herd level and variation of heritability

For studying the genotype by environment interaction on milk production we considered, according to FALCONER'S theory (1952) the expression of the same trait in two environments as two different, but genetically related traits. The data of the french milk recording file are divided according to farm environmental effect into three groups to which we associated three 
different environments, by hypothesis. The heritability of fat and protein yield increased with the production level $(0.31,0.33$ and 0.42$)$. The genetic correlations between environments were close or larger than 0.9 . These findings, which confirm those of the many studies already made in that field, allow to draw some conclusions about dairy cattle selection in France. The choice of bulls on a single estimated breeding value is sufficient whatever the production level of the farms. It is not necessary to set up specialized strains for each production level. The increase in selection efficiency, which could be obtained if progeny testing were only made in farms with a high production level, would be cancelled by the effects of the reduction in progeny testing facilities. Accordingly, progeny testing, should still be made in all farms.

\section{Références bibliographiques}

AverdunK G., AlPS H., 1971. Die Interaktion zwischen Herdenniveau und vater bei der Milchleistung des Fleckviehs in Bayern. Z. Tierz. Zuchtbiol., 88, 197-202.

BONAITI B., BERTAUdière L., 1982. Interaction génotype milieu sur la production laitière chez les bovins 1. Variation de la production laitière des primipares sous l'influence de l'indice de sélection du père et du niveau d'étable. Ann. Génét. Sél. anim., 1982, 14, 177-186.

BurdicK J.M., Mc Guilliard L.D., 1963. Interaction between sires in artificial insemination and management of dairy herds. J. Dairy Sci., 46, 452-458.

Branton C., Rios G., Evans D.L., Farthing B.R., Kodoce K.L., 1974. Genotype climatic and other interactions effects for productive responses in Holsteins. J. Dairy Sci., 57, 833-841.

Burnside E.B., RENNIE J.C., 1968. Sire evaluation and herd level of milk production. Can. J. Anim. Sci., 48, 97-102.

DANELL B., 1982. Interaction between genotype and environment in sire evaluation for milk production. Acta Agric. scand., 32, 33-44.

FALCONER D.S., 1952. The problem of environment and selection. Amer. Nature, 86, 293-298.

GraverT H.O., 1958. Untersuchungen uber die Heritabilitat der butterfbtleistung. Z. Tierz. Zuchtb., 71, 155-163.

GRAVERT H.O., 1959. Zur Bewertung der milchfettleistung in absoluten oder prozentischen Zahlen. Zuchtungskunde, 31, 345-349.

HENDERSON C.R., 1953. Estimation of variance and covariance components. Biom., 9, 226-252.

LEGATES J.E., 1962. Heritability of fat yields in herds with different production levels. J. Dairy Sci., 45, 990-993.

LYTTON V.H., LEGATES J.E., 1966. Sire by region interaction for production traits in dairy cattle. J. Dairy Sci., 49, 874-877.

Mc Daniel B.T., CORLey E.L., 1967. Relationship between sire evaluation at different Herdmate levels. J. Dairy Sci., 50, 735-741.

Mc Dowell R.E., Wiggans G.R., Camoen J.K., Van Vleck L.D., Saint-Louis D.G., 1976. Sire comparisons for Holsteins in Mexico versus the United States and Canada. J. Dairy Sci., 59, 298-304.

Mallard J., Masson J.-P., Douaire M., 1983. Interaction et modèle mixte. 2. Statistique pour un modèle incluant un niveau de hiérarchie. (A paraître).

MAO I.L., BURNSIDE E.B., 1969. Sire by herd environment interaction for milk production. J. Dairy Sci., 52, 1055-1062.

MASON I.L., ROBERTSON A., 1956. The progeny testing of dairy bulls at different levels of production. J. Agricult. Sci., 47, 367-375.

Mitchell R.G., CoRley E.L., TYler W.J., 1961. Heritability, phenotypic and genetic correlations between type ratings and milk and fat production in Holstein-Friesian cattle. J. Dairy Sci., 44, 1502-1509.

Norman H.D., Mc Daniel B.T., Dickinson F.N., 1972. Conflicts between heritability estimates of mature equivalent and herdmate-deviation milk and fat. J. Dairy Sci., 55, 1, 507-517.

PETERSEN P.H., 1975. Genotype environment interaction in milk production under Danish and Bulgarian. Czechoslovakian conditions. Anim. Prod., 21, 101-108. 
Poutous M., Briend M., Calomiti S., Doan D., Felgines C., Steier G., 1981. Méthode de calcul des index laitiers. Bases générales (1). Bull. tech. Inf., 361, 433-446.

Richardson D.O., OWen J.R., Plowman R.D., Miles J.T., 1971. Importance of sire $\times$ ration interactions in production and feed intake traits of dairy cattle. J. Dairy Sci., 54, 1518-1525.

Robertson A., O'ConNor L.K., EDWards J., 1960. Progeny testing dairy bulls at different management levels. Anim. Prod., 2, 141-152.

SYRSTAD O., 1966. Study on dairy herd records. IV. - Estimates of phenotypic and genetic parameters. Acta Agric. scand., 16, 79-96.

VAN VLECK L.D., 1963. Genotype and environment in sire evaluation. J. Dairy Sci., 46, 983-987.

VAN VLECK L.D., BRADFORD G.E., 1964. Heritability of milk yield at different environmental levels. Anim. Prod., 6, 285.

Van Vleck L.D., Wadeu L.H., Henderson C.R., 1961. Components of variance associated with milk and fat records of artificially sired Holstein daughters. J. Anim. Sci., 20, 812-816.

WIGGANS G.R., VAN VLECK L.D., 1970. Sire effects in different housing systems. J. Dairy Sci., 53, 545-547.

WIGGANS G.R., VAN VLECK L.D., 1978. Evaluation of sire in herds feeding differing proportions of concentrates and roughages. J. Dairy Sci., 61, 246-249. 